Check for updates

Cite this: RSC Adv., 2017, 7, 53401

Received 28th September 2017 Accepted 2nd November 2017

DOI: 10.1039/c7ra10757a

rsc.li/rsc-advances

\section{Improved shellac mediated nanoscale application drug release effect in a gastric-site drug delivery system $\dagger$}

\begin{abstract}
$\mathrm{Ke} \mathrm{Ma},{ }^{a}$ Yiping Qiu, ${ }^{b}$ Yaqin $\mathrm{Fu}^{\mathrm{c}}$ and Qing-Qing $\mathrm{Ni}$ (DD *de
The development of biocompatible nanocomposites for biomedical applications such as drug release has attracted increasing attention in recent years. Shellac/sodium shellac nanofibers and nanoparticles were produced for drug delivery applications. Ketoprofen was loaded on the drug delivery system (DDS), and three schemes based on shellac/sodium shellac were chosen. The biocompatibility and nontoxicity of shellac and the promising results of the nanofibers and nanoparticles highlighted their challenging potential for controlled drug delivery applications. A significant increase of drug release rate can be observed at the gastric site. The prepared drug-loaded nanofibers and nanoparticles were characterized by scanning electron microscopy (SEM), Fourier transform infrared spectroscopy (FTIR) and X-ray diffraction (XRD). The results show that the above-mentioned drug delivery system is able to support high-efficiency encapsulation. A new drug delivery system was designed and studied through shellac and sodium shellac. The application scope of shellac to DDSs is extended.
\end{abstract}

\section{Introduction}

Nanotechnology drug delivery system (DDS) research has attracted the attention of pharmaceutical, biotechnology, and healthcare industries during the recent decades. ${ }^{1-5}$ As the nanotechnology has been developed during the decades with several historical breakthroughs, ${ }^{6-11}$ numerous new applications have been applied to DDSs. ${ }^{12-14}$ Nanoparticles and nanofibers have been widely used in DDSs due to their popular and simple production methods. Drug delivery system characteristics are improved with the incorporation of biodegradable polymer carriers which sustain the release of encapsulated drugs and reduce the side effects. For a viable DDS, a drug carrier needs to overcome several major problems such as loading capacity for large amounts of drugs, controlled release profile of therapeutics at the targeted location, non-toxicity, biodegradability and cost effectiveness. Nano DDS technology, such as nano-formulated drugs, drug eluting stents,

\footnotetext{
${ }^{a}$ Interdisciplinary Graduate School of Science and Technology, Shinshu University, Ueda 386-8567, Japan. E-mail: 16st107g@shinshu-u.ac.jp

${ }^{b}$ Department of Technical Textiles, College of Textiles, Donghua University, Shanghai 201620, China.E-mail: ypqiu@dhu.edu.cn

${ }^{c}$ Key Laboratory of Advanced Textile Materials and Manufacturing Technology, Ministry of Education, Zhejiang Sci-Tech University, Hangzhou 310018, China. E-mail:fyq01@zstu.edu.cn

${ }^{d}$ Department of Mechanical Engineering \& Robotics, Shinshu University, Ueda 3868567, Japan. E-mail: niqq@shinshu-u.ac.jp

${ }^{e}$ College of Textile and Garments, Anhui Polytechnic University, Wuhu, Anhui 241000, China

$\dagger$ Electronic supplementary information (ESI) available: SEM images are provided. See DOI: $10.1039 / \mathrm{c} 7 \mathrm{ra} 10757 \mathrm{a}$
}

drug coatings and devices, involves improved efficacy, targeted drug delivery, reduced active drug ingredients and reduced drug side effects. This technology is able to enhance the efficacy and sustain drug release. It can also promote the business value of the healthcare applications. The main aim of the nano-formulations is to adjust the normal metabolic profile of proven established drug molecules by significantly improving the drug efficacy, sustaining release and reducing side effects simultaneously. ${ }^{\mathbf{1 5}}$ Nanotechnology applications based on DDSs include nanoemulsions, nanoparticles, liposomes and nanofibers. Nanoproduct drug delivery systems (DDSs) have the advantages of lower cost, scalability, targeted delivery, reduced side effects, biodegradability, biocompatibility, sustainability in release of encapsulated drug and improved efficacy. ${ }^{16}$ In this work, nanofibers and nanoparticles were prepared to fine-tune the release speed by their different drug effects. Shellac is always used in colon DDS due to the very poor drug effect in gastric environment. A simple method was employed to improve the shellac drug release effect in gastric environment to expand the scope of shellac carrier in vivo environment DDS. Less drug wastage was observed in this new DDS than the formerly ones.

The major difference between electrospinning and electrospray lies in their working medium. The electrospray process adopts a low viscosity solution while the electrospinning process adopts a high viscosity solution. Electrospray is the most common molding method to obtain monodisperse particles. ${ }^{17,18}$ Electrospinning is a nanoscale fiber production method employing the electric force to draw charged threads of polymer solutions, which results in surface deformation of the charged liquid. ${ }^{19-22}$ 
The elongation of the liquid resulting from this bending instability leads to the formation of uniform nanofibers. ${ }^{23}$ Electrospinning processes characteristics of both electrospray and dry spinning of fibers. ${ }^{24}$ The in-depth study of electrospinning involves electrostatic, electrohydrodynamics, rheology, aerodynamics and other fields. ${ }^{25-27}$

Shellac has a chemical structure which shown in Fig. 1. Shellac, also referred to as lac, is a kind of resin secreted by the female lac bug on trees in the forests of India and Thailand. Refined shellac can be made by rinsing and filtration of crude shellac which is directly secreted by lac bug. Shellac has been widely used in food, medicine, military, coating, dyes and other industries fields due to its excellent properties of film-forming, waterproof, fixability and biocompatibility. In the pharmaceutical industry, shellac was originally used in the coating of the enteric drug. However, due to the emergence of various synthetic materials since the 1950s gradually, shellac faded out of their original role in various fields. But with the development of biomedical science, increasing attention has been paid to the limitations of synthetic materials, such as irritation, carcinogenicity, etc. The application of shellac has been extended to DDS, since it is the only animal-secreted resin that can be used in pharmaceutically applications. ${ }^{28,29}$

In this study, shellac carrying drug effect was improved by alkalization method adopting sodium shellac through in gastric environment. Shellac's acid groups are not easy to dissociate because of its high acid dissociation constant $\left(\mathrm{p} K_{\mathrm{a}}\right)$. This results in a poor drug effect of shellac in acid fluid environment $(\mathrm{pH} 2)$ and there is a limitation of release rate at only about $10 \%$. In this study, a one-step chemical modification of the shellac is carried out by weak alkaline sodium carbonate solution. By adopting this one-step chemical modification, better degradation performance was observed in simulating gastric fluid environment. The drug effect of shellac in intestinal fluid was also improved. Although extensive study has been done on shellac nanofiber in colon DDS, little literature is available on the synthesis of sodium shellac and its composite with shellac as drug carrier in gastric environment. Therefore, this study on nanofiber and nanoparticle mats composed of shellac/sodium shellac loaded with ketoprofen (Fig. 2) as a guest drug is carried out. For optimization, three ratios of shellac/sodium shellac mixing schemes were used. The results obtained from scanning electron microscope (SEM), Fourier transform infrared spectroscopy (FTIR) and X-ray diffraction (XRD) and drug release analysis confirmed the nanocomposite

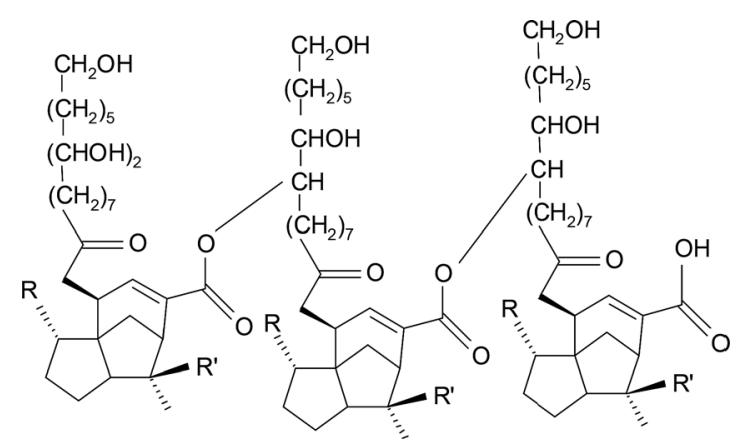

Fig. 1 Chemical structure of shellac.<smiles>CC(C(=O)O)c1cccc(C(=O)c2ccccc2)c1</smiles>

Fig. 2 Chemical structure of ketoprofen.

mat to be suitable for the controlled release of ketoprofen for biomedical applications with a tunable release profile.

Thanks to excellent biocompatibility and nontoxicity of shellac and sodium shellac, the corresponding nanocomposite can be considered as a viable drug carrier medium. And hence, it can be applied in drug delivery systems, tissue engineering and other biomedical applications. Further study on shellac/ sodium shellac nanocomposites can be carried out to extend its application to biomedical use.

\section{Experimental}

\subsection{Materials}

The wax free shellac used in this study was provided by Kigata, Japan. Ketoprofen $5 \mathrm{~g}\left(M_{\mathrm{w}}=254.28\right)$, phosphate buffered saline (PBS, 1/15 mol L ${ }^{-1}$ with $\mathrm{pH}$ 6.8), hydrochloric acid ( $\mathrm{HCl}$ ), sodium carbonate and ethanol were purchased from SigmaAldrich (Japan) or Wako Pure Chemicals (Japan). All chemicals were used without further purification. Water was doubledistilled just before use.

\subsection{Preparation of sodium shellac}

Sodium shellac (shellac-COONa) was obtained by neutralization reaction of shellac and sodium carbonate solution. $5 \mathrm{~g}$ shellac was added every half hour into $50 \mathrm{~mL}, 0.1 \mathrm{~mol} \mathrm{~L}^{-1}$ sodium carbonate solution during magnetic stirring until it was no longer dissolved, the temperature was kept at $40{ }^{\circ} \mathrm{C}$ to speed up the reaction. No gas was observed during the neutralization process. And there was no weight lost after the neutralization process. Then the sodium shellac was obtained after drying 48 hours under room temperature.

\subsection{Shellac/sodium shellac solution preparation}

A mixed solution composed of $65 \%(\mathrm{w} / \mathrm{v})$ of shellac and $5 \%(\mathrm{w} / \mathrm{v})$ ketoprofen in ethanol were mixed with solvent formulation for an optimal electrospinning solution to make NF1. 40\% (w/v) shellac and $5 \%(\mathrm{w} / \mathrm{v})$ ketoprofen for electrospray fluid to obtain NP1. $32.5 \%(\mathrm{w} / \mathrm{v})$ of shellac, $32.5 \%(\mathrm{w} / \mathrm{v})$ of sodium shellac and $5 \%(\mathrm{w} / \mathrm{v})$ ketoprofen in ethanol to prepare NF2. 20\% $(\mathrm{w} / \mathrm{v})$ of shellac, $20 \%(\mathrm{w} / \mathrm{v})$ of sodium shellac and $5 \%(\mathrm{w} / \mathrm{v})$ ketoprofen in ethanol to prepare NP2. 65\% (w/v) of sodium shellac and $5 \%(\mathrm{w} / \mathrm{v})$ ketoprofen in ethanol to prepare NF3. 65\% $(\mathrm{w} / \mathrm{v})$ of sodium shellac and $5 \%(\mathrm{w} / \mathrm{v})$ ketoprofen in ethanol to prepare NP3. These six parameters were investigated in three types of nanoparticles and three types of nanofibers. The resultant nanofibers and nanoparticles were collected on a metal collector wrapped with aluminum foil at affixed distance of $15 / 20 \mathrm{~cm}$ from the needle tip of the spinning/spray 
head. Their average sizes were determined by measuring the diameters of more than 100 particles in SEM images using the Image J software (National Institutes of Health, USA). Details of the parameters for electrospinning, electrospray process and the resultant products are listed in Table 1.

\subsection{Electrospinning and electrospray process}

For electrospinning and electrospray process, an electrospinning apparatus manufactured by Kato Tech Co. Ltd. (Kyoto, Japan) was used. The applied voltage for both electrospinning and electrospray process was $15 \mathrm{kV}$. The distance from needle tip to collector was $20 \mathrm{~cm}$ for electrospinning process and for electrospray process was $15 \mathrm{~cm}$.

\subsection{Drug release test}

The drug releases characteristics of the prepared samples were investigated by immersing all nano-composites into $50 \mathrm{~mL}$ $0.01 \mathrm{~N} \mathrm{HCl}(\mathrm{pH} 2)$ for 24 hours and PBS ( $\mathrm{pH} 6.8$ ) respectively and thermostatically shaken $(100 \mathrm{rpm})$ for 24 hours at $36{ }^{\circ} \mathrm{C}$. To determine the drug concentration, $4 \mathrm{~mL}$ of the test solution was taken at particular time intervals; $4 \mathrm{~mL}$ of new test solution was then added for capacity adjustment. A UV visible spectro photometer (V-530, JASCO, Japan) was used to determine the concentration of the ketoprofen released from collected test solution. To determine the concentration of ketoprofen, a calibration curve from the ultraviolet-visible absorption spectrum of ketoprofen was prepared in advance.

\subsection{Characterization}

The morphology of the electrospinning nanofibers and electrospray nanoparticles were investigated by SEM-EDS (Hitachi 3000H SEM).

For a morphological study of the nanofibers and nanoparticles. FTIR analysis (IR Prestige 21, Shimadzu, Japan) was carried out to study chemical structure of the surface of the prepared nanofibers and nanoparticles. In addition, the test temperature was maintained at $36{ }^{\circ} \mathrm{C}, 100 \mathrm{rpm}$ using a Shaking incubator (PIC-100S, Askul, Japan). X-ray diffraction (XRD) was performed on a $\mathrm{D} /$ Max-BR diffractometer (RigaKu, Tokyo, Japan) over the $2 \theta$ range of $5-80^{\circ}$ using $\mathrm{CuK} \alpha$ radiation at $40 \mathrm{mV}$ and $30 \mathrm{~mA}$.

Table 1 Parameters used for electrospinning and electrospray process $^{e f}$

\begin{tabular}{llll}
\hline Number & Solution ingredient & Distance $(\mathrm{cm})$ & Diameter (nano-meter) \\
\hline $\mathrm{NF}^{a} 1$ & $\mathrm{~S}^{c} 100 \%$ & 20 & $512 \pm 250$ \\
$\mathrm{NF} 2$ & $\mathrm{~S}: \mathrm{SS}^{d} 50 \%: 50 \%$ & 20 & $448 \pm 250$ \\
$\mathrm{NF} 3$ & $\mathrm{SS} 100 \%$ & 20 & $351 \pm 250$ \\
$\mathrm{NP}^{b} 1$ & $\mathrm{~S}^{c} 100 \%$ & 15 & $699 \pm 750$ \\
$\mathrm{NP} 2$ & $\mathrm{~S}: \mathrm{SS} 50 \%: 50 \%$ & 15 & $526 \pm 600$ \\
$\mathrm{NP} 3$ & $\mathrm{SS} 100 \%$ & 15 & $345 \pm 600$
\end{tabular}

${ }^{a}$ NF: nanofiber. ${ }^{b}$ NP: nanoparticle m. ${ }^{c}$ Shellac. ${ }^{d}$ Sodium shellac. ${ }^{e}$ All tips with inner diameter of $0.6 \mathrm{~mm} .{ }^{f}$ The shell fluid consisted of $5 \%$ $(w / v)$ ketoprofen in ethanol solution.

\section{Results and discussion}

\subsection{Characterization of sodium shellac}

After the $\mathrm{Na}_{2} \mathrm{CO}_{3}$ solution treatment, it can be observed that the colour of sodium shellac changed from yellow to brown as shown in Fig. 3. No gas release was observed during the reaction process and no weight were lost during the process.

3.1.1 FTIR analysis. The FTIR of sodium and shellac sodium, ATR-FTIR were conducted to investigate the compatibility between the shellac and the shellac sodium salt. It can be observed from Fig. 4 that the basic trends of sodium shellac and shellac are similar, indicating that the change in the general structure is not very significant of shellac sodium salt relative to the shellac. But the shellac sodium has a characteristic at $1560 \mathrm{~cm}^{-1}$.

The appearance of this characteristic peak shows the difference between the sodium shellac and shellac, it also proves the presence sodium shellac meanwhile.

The characteristic peak at $1560 \mathrm{~cm}^{-1}$ is because after the formation of the carboxylic acid salt, the carboxylic acid anion occurred resonance effect and produced coupling effect.

3.1.2 XRD analysis. No distinct peaks in the spectrum of shellac sodium and shellac evidently indicated in Fig. 5 that the molecular orientation and arrangement of the polymers were disordered, i.e., an amorphous state. Similarly, there were no discrete peaks in the spectrum of nanofiber and nanoparticle from curve $\mathrm{a}$ and $\mathrm{b}$ which implied that the crystalline structure cannot be changed no matter it is in the nanofiber or in the nanoparticle part. And there were no discrete peaks in the spectrum of shellac and sodium shellac nanofibers and nanoparticles too. It indicates that in the nanomaterials, shellac and sodium shellac salt existed as the amorphous state in the both nanofiber and nanoparticle forms.

\subsection{SEM analyses morphology of shellac and sodium shellac nanofibers \& nanoparticles}

Three kinds of shellac/sodium shellac/ketoprofen nanofibers and three kinds of nanoparticles have been observed respectively in Fig. $7 \mathrm{a}$ and $\mathrm{b}$.

Different sizes of extreme distribution nanoparticles can be observed. It is because the droplets tend into ellipsoid or irregular spherical in the role of gravity deformation. Larger charge density occurs in distribution on the sphere surface is heterogeneity. There is larger charge density in regions with larger curvatures. This caused the droplets affected by different directions and uneven forces. Different sizes of extreme distribution nanoparticles were generated during the division

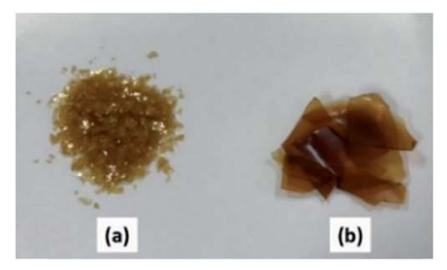

Fig. 3 Photo of shellac (a) and sodium shellac (b). 


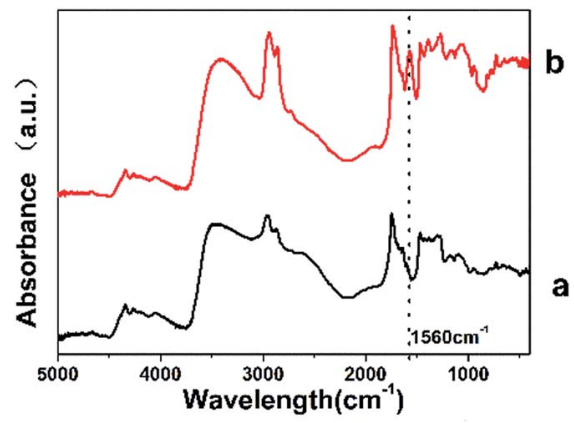

Fig. 4 FTIR spectra of shellac (a) and sodium shellac (b).

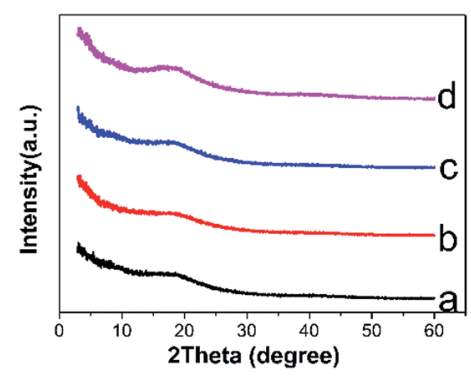

Fig. 5 XRD spectra of shellac nanofiber (a), shellac nanoparticle (b), sodium shellac nanofiber (c), sodium shellac nanoparticle (d).

process of droplets. This phenomenon has more significant effect when the molecular mass is greater. The diameter distribution in NP1 is the most uneven one for this reason.

Table 1 lists the details of shellac and sodium shellac resulted in nanofibers in which NF1, 2, 3 were obtained by electrospinning whilst NP1, 2, 3 were obtained by electrospray method (Fig. 7b). It can be found that the average diameters of both nanofiber and nanoparticle will be finer if more sodium shellac was added as shown in Fig. $7 \mathrm{a}$ and d.

The photographs of nanofibers and nanoparticles mats that evaluated in Fig. 7a and b are obtained after $15 \mathrm{~min}$ of electrospinning/electrospray process to detect the macroscopic difference. A light yellow appears on nanofibers at room temperature while darker yellow can be observed on nanoparticles. As all of the nanofibers and nanoparticles have bigger than $100 \mathrm{~nm}$ (bigger than the wavelength of the light). This phenomenon may because nanoparticle mats have a bigger density than nanofiber.

\subsection{FTIR evaluations of shellac and sodium shellac nanofibers and nanoparticles}

The FTIR spectra of shellac and sodium shellac nanofibers and nanoparticles are illustrated in Fig. 6a and b respectively. For all samples, the characteristic peaks of shellac can be observed at $1710 \mathrm{~cm}^{-1}, 2860 \mathrm{~cm}^{-1}$ and $2930 \mathrm{~cm}^{-1}$. In addition, sodium shellac characteristic peak can be observed at $1560 \mathrm{~cm}^{-1}$ as shown in NF 3 and NP3. The rate of sodium shellac content shows a proportional relationship in the curve which prove the exit of sodium shellac. In the case of shellac/ketoprofen-sodium
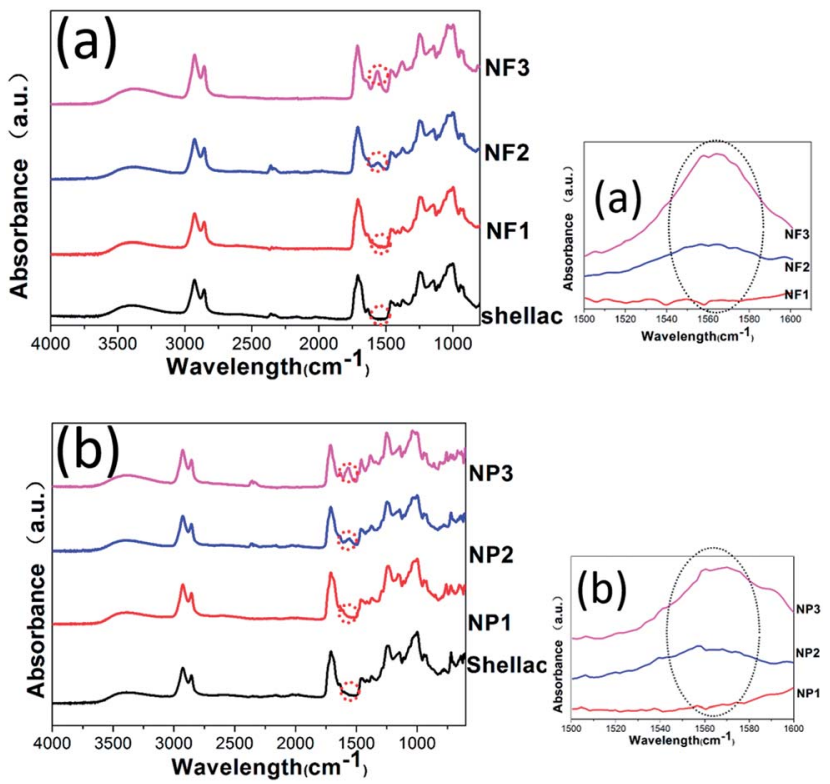

Fig. 6 (a) FTIR spectrum of drug filled shellac nanofiber composite with sodium shellac with 0\% (NF1), 50\% (NF2) and 100\% (NF3). (b) FTIR spectrum of drug filled shellac nanofiber composite with sodium shellac with 0\% (NP1), 50\% (NP2) and 100\% (NP3).

shellac, the characteristic peaks of ketoprofen overlapped completely with the absorption bands of shellac and sodium shellac so that these bands are unavailable for differentiation. The FTIR results show that there is no difference in chemical structure between nanofiber and nanoparticle.

\subsection{Functional performance and controlled-release mechanism}

In the vitro drug release profiles of the three kinds nanofibers and nanoparticles are given in Fig. 8a and b. In the acidic conditions of pH 2 (Fig. 8a), the release amount of the shellac/shellac sodium nanoscale application cannot reach to $100 \%$ as that in $\mathrm{pH} 6.8$ dissolution medium. Agglomeration of the carrier can be observed in the bottom of the bottle. This is mainly because, at low pH conditions, the acid ions in the shellac combine with high concentrations of oxygen ions. The drug release behaviors are also studied in colon situation (pH 6.8) to keep the integrity of drug release in vitro. The sodium shellac has larger drug effect than pure shellac. The nanoparticles NP1 release entirely instantly when they were put into the $\mathrm{pH} 6.8$ medium.

The drug release effect of the sodium shellac is larger and drug release rate is quicker than pure shellac, whereas the drug release effect of nanoparticle is larger and drug release rate is higher than nanofiber. It is because the specific surface area of nanoparticle is obviously bigger than nanofiber.

In addition, sodium shellac nanoscale applications (NF3, NP3) can be proved with good biodegradability because they can be observed almost totally dissolved after drug release process in pH 6.8 PBS solution. In the same condition, NF1/NP1 can not be observed dissolved obviously and NF2/NP2 can be observed part dissolved. The drug release effect of the sodium shellac is 
(a)
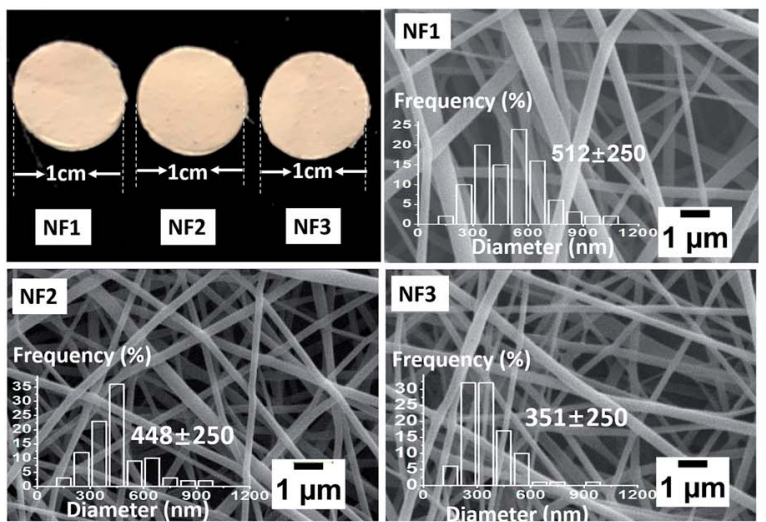

(b)
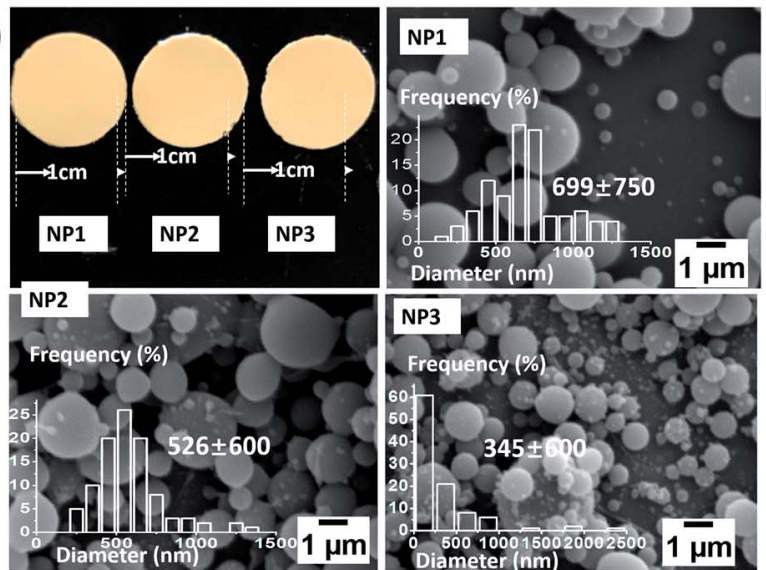

Fig. 7 (a) Photographs, SEM images and diameter distribution of shellac/sodium shellac nanofiber composite sodium shellac with $0 \%$ (NF1), 50\% (NF2) and 100\% (NF3). (b) Photographs, SEM images and diameter distribution of shellac/sodium shellac nanofiber composite sodium shellac with 0\% (NP1), 50\% (NP2) and 100\% (NP3).

improved mainly through the principle of neutralization reaction. The functional groups at extremity of shellac molecule are not easy to dissociate. In sodium shellac, they changed to carboxylate radical which are easier ionized in water.

The aforementioned reasons make sodium shellac can be dissolved easier at lower $\mathrm{pH}$ conditions. In the same simulated environment with the $\mathrm{pH}$ value of the intestinal juice, the drugloaded film of the sodium salt of the shellac showed a good release effect. Some studies carried out by other researchers are further discussed below. Wang et al. ${ }^{29}$ synthesized shellac nanofiber for colon-targeted drug delivery. The researchers claimed a very small percentage of drug release at $\mathrm{pH}$ 2.0. They claimed the preparation of shellac nanofiber a release rate $8.2 \%$ and 9.3\% during the first two hours at pH 2.0 PBS. And around $40 \%$ drug was released after 30 min immersion in pH 6.8 PBS. This result provided very similar sustained release profiles with our study for shellac nanofiber. The study did not report a further study for the suitability of the shellac nanoparticles and sodium shellac for drug delivery. Comparing the study of Wang et al. ${ }^{29}$ and Cui et al. ${ }^{30}$ pure shellac nanofibers and shellac nanoparticles were respectively analyzed for drug delivery in vitro. Both of their whole in vitro dug release processes (pH 2 and $\mathrm{pH}$ 6.8) were only last for about 10 hours. They (a)

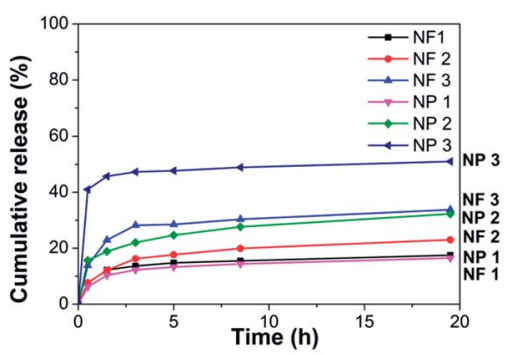

(b)

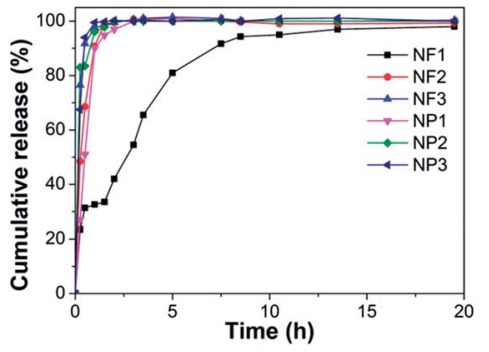

Fig. 8 (a) Cumulative release from shellac nanofiber/nanoparticle composite with sodium shellac with 0\% (NF 1, NP1), 50\% (NF2, NP2) and $100 \%$ (NF3, NP3) in $\mathrm{pH}$ 2. (b) Cumulative release from shellac nanofiber/nanoparticle composite with sodium shellac with 0\% (NF1, NP1), 50\% (NF2, NP2) and 100\% (NF3, NP3) in pH 6.8.

claimed the preparation of shellac nanofiber/nanoparticle a release rate under $10 \%$ during the first two hours at $\mathrm{pH} 2.0$ environment. And the drug release in gastric environment almost stop within 2 hours. While in our study, the release rate effect improved to $50 \%$ and lasting 20 hours in gastric environment.

In another study, Luo et al. ${ }^{31}$ successfully developed sodium shellac particles for wrapping disperse multiscale emulsion. Sodium shellac has been reported as a novel wrapping material that can aggregate and wrap emulsion micelles driven by different forces. However, due to the high solid content of the nanoscale emulsion particles, they are difficult to wrap by the spray-drying method. Electrospray may be used as an easier way to produce shellac nanoparticles.

\section{Conclusions}

This study reported a novel method of shellac synthesis in the suitable utilization of a natural animal secreted resin. A potential to develop capsule form applications which can remain in the stomach for a long time. The sodium shellac which proved a larger and quicker drug effect in gastric environment. The sodium shellac was mixed in three concentrations with shellac $(0 \%, 50 \%$ and $100 \%)$ and then electrospinning/electrospray into a composite mat. Nanofibers and nanoparticles loaded with ketoprofen were prepared successfully for sustained drug release. The results revealed that the release speed increased with the further addition of sodium shellac. And nanoparticle shows better drug effect than nanofiber. Therefore, the results show that controlled drug release profile can be achieved by tuning sodium shellac in nanofiber/ nanoparticle composite. Thus, it is confirmed that the sodium 
shellac/shellac composite as a sustained drug delivery nanofiber/nanoparticle mat.

\section{Conflicts of interest}

There are no conflicts to declare.

\section{Acknowledgements}

I would like to express my sincere gratitude to my supervisor, Professor Qing-Qing Ni, for his instructive advices on my work. Then special thanks extend to my dear sweet Lubin Qu, who helped me correct my paper amidst his busy schedule and encouraged me. I am also deeply indebted to all the staff in Shinshu University Global Leader Program for Fiber Renaissance for their long-lasting support and encouragement.

\section{References}

1 Y. Zhu, J. Ding, Y. Fu and Y. Dong, Polym. Compos., 2016, 37, 1728-1734.

2 L. Yu, Y. Zhu, C. Qian, Q. Fu, Y. Zhao and Y. Fu, J. Nanomater., 2016, 1, 1-8.

3 Y. Wang, Y. Zhu, X. Fu and Y. Fu, Text. Res. J., 2015, 85, 980986.

4 Q.-Q. Ni, Y.-F. Zhu, L.-J. Yu and Y.-Q. Fu, Nanoscale Res. Lett., 2015, 10, 174.

5 Y.-F. Zhu, Q.-Q. Ni and Y.-Q. Fu, RSC Adv., 2015, 5, 37483756.

6 Y. Dong, Q.-Q. Ni, L. Li and Y. Fu, Mater. Lett., 2014, 132, 206209.

7 J. Ding, Y. Zhu and Y. Fu, Polym. Compos., 2014, 35, 412-417. 8 X. Fu, J. Wang, J. Ding, H. Wu, Y. Dong and Y. Fu, Compos. Sci. Technol., 2013, 87, 170-173.

9 Y. Dong, J. Ding, J. Wang, X. Fu, H. Hu, S. Li, H. Yang, C. Xu, M. Du and Y. Fu, Compos. Sci. Technol., 2013, 76, 8-13.

10 Y. Dong, R. Wang, S. Li, H. Yang, M. Du and Y. Fu, J. Colloid Interface Sci., 2013, 391, 8-15.

11 X. Jin, Q. Q. Ni, Y. Fu, L. Zhang and T. Natsuki, Polym. Compos., 2012, 33, 317-323.
12 J. K. Vasir, K. Tamb wekar and S. Garg, Int. J. Pharm., 2003, 255, 13-32.

13 E.-R. Kenawy, F. I. Abdel-Hay, M. H. El-Newehy and G. E. Wnek, Mater. Chem. Phys., 2009, 113, 296-302.

14 M. Ke, J. A. Wahab, B. Hyunsik, K.-H. Song, J. S. Lee, M. Gopiraman and I. S. Kim, RSC Adv., 2016, 6, 4593-4600. 15 R. Sridhar and S. Ramakrishna, Biomatter, 2013, 3, e24281.

16 M. Siebers, X. Walboomers, S. Leeuwenburgh, J. Wolke and J. Jansen, Biomaterials, 2004, 25, 2019-2027.

17 L. Reyderman and S. Stavchansky, Int. J. Pharm., 1995, 124, 75-85.

18 C. Bitz and E. Doelker, Int. J. Pharm., 1996, 131, 171-181.

19 R. Falk, T. W. Randolph, J. D. Meyer, R. M. Kelly and M. C. Manning, J. Controlled Release, 1997, 44, 77-85.

20 I. Genta, M. Costantini, A. Asti, B. Conti and L. Montanari, Carbohydr. Polym., 1998, 36, 81-88.

21 C. Berkland, K. K. Kim and D. W. Pack, J. Controlled Release, 2001, 73, 59-74.

22 C. Berkland, D. W. Pack and K. K. Kim, Biomaterials, 2004, 25, 5649-5658.

23 J. Zeleny, Phys. Rev., 1914, 3, 69.

24 J. Fenn, Science, 1989, 246, 64.

25 Z. K. Nagy, A. Balogh, G. Drávavölgyi, J. Ferguson, H. Pataki, B. Vajna and G. Marosi, J. Pharm. Sci., 2013, 102, 508-517.

26 W. Li, D.-G. Yu, K. Chen, G. Wang and G. R. Williams, Mater. Lett., 2013, 93, 125-128.

27 Z. K. Nagy, I. Wagner, Á. Suhajda, T. Tobak, A. Harasztos, T. Vigh, P. Soti, H. Pataki, K. Molnar and G. Marosi, eXPRESS Polym. Lett., 2014, 8, 5.

28 S. Limmatvapirat, D. Panchapornpon, C. Limmatvapirat, J. Nunthanid, M. Luangtana-Anan and S. Puttipipatkhachorn, Eur. J. Pharm. Biopharm., 2008, 70, 335-344.

29 X. Wang, D.-G. Yu, X.-Y. Li, S. A. Bligh and G. R. Williams, Int. J. Pharm., 2015, 490, 384-390.

30 L. Cui, Z.-P. Liu, D.-G. Yu, S.-P. Zhang, S. A. Bligh and N. Zhao, Colloid Polym. Sci., 2014, 292, 2089-2096.

31 Q. Luo, K. Li, J. Xu, K. Li, H. Zheng, L. Liu, H. Zhang and Y. Sun, J. Agric. Food Chem., 2016, 64, 9374-9380. 